\title{
Educación Ambiental en la Institución Educativa Luis Rodríguez Valera, en el municipio de Valledupar, Cesar
}

\author{
Environmental Education at the Luis Rodríguez Valera \\ Educational Institution, in the municipality of \\ Valledupar, Cesar
}

\section{Educação ambiental na Instituição Educativa Luis Rodríguez Valera, no município de Valledupar, Cesar}

\author{
Juan Carlos Rivera Mendoza \\ Magister en Desarrollo Sostenible y Medio Ambiente \\ Universidad de Manizales \\ juancarlosriveramendoza02@gmail.com
}

\section{Resumen}

La presente investigación denominada Estado de la educación ambiental en la institución educativa Luis Rodríguez Valera, en el municipio de Valledupar, Cesar, pretendió desarrollar lineamientos de educación ambiental en la Institución Educativa Luis Rodríguez Valera, en el municipio de Valledupar, Cesar. Teóricamente estuvo sustentada en los postulados de la Conferencia del Tbilisi (1977), Declaración de Río sobre el Medio Ambiente y el Desarrollo (1993), Congreso Internacional Unesco PNAMU sobre Educación Formación y Formación Ambiental Moscú (1987), La Declaración de Comodoro Rivadavia. Transformar Nuestro Mundo: la Agenda 2030 para el Desarrollo Sostenible (Sauvé, 2014), además de las Normativas Legales de Colombia. Metodológicamente se enmarcó en el enfoque multimétodo y aplicada. Su desarrollo se estructuró en seis trayectorias. La población estuvo conformada por trecientos ochenta y cinco (385) estudiantes, un (1) Rector, diecinueve (19) docentes. Con una muestra no probabilística de noventa (90) estudiantes, diez (10) docentes y un grupo focal de siete (7) estudiantes. Con el análisis de los resultados de las entrevistas aplicadas a la muestra del estudio se consideró que en la Institución Educativa Luis Rodríguez Valera hay muy poca conciencia ecológica, por lo que fue necesario construir la propuesta Unidos cuidaremos de nuestra casa, la Tierra que luego de aplicada y sistematizados sus resultados. Se concluyó que su ejecución fue exitosa, logrando la formación de la conciencia ecológica tanto en los estudiantes como en los maestros.

Palabras clave: Estrategias, educación ambiental, conciencia ecológica. 


\begin{abstract}
The present investigation called State of the environmental education in the educational institution Luis Rodriguez Valera, in the municipality of Valledupar, Cesar pretended to develop environmental education guidelines in the Educational Institution Luis Rodríguez Valera, in the municipality of Valledupar, Cesar. Theoretically it was supported by the postulates of the Tbilisi Conference (1977), Rio Declaration on Environment and Development (1993), Unesco International Conference PNAMU on Education Training and Environmental Training Moscow (1987), The Declaration of Comodoro Rivadavia. Transforming Our World: the 2030 Agenda for Sustainable Development (Sauvé, 2014), in addition to the Legal Norms of Colombia. Methodologically it was framed in the multimethod and applied approach. Its development was structured in six trajectories. The population consisted of three hundred and eighty-five (385) students, one (1) Rector, nineteen (19) teachers. With a non-probabilistic sample of ninety (90) students, ten (10) teachers and a focus group of seven (7) students. With the analysis of the results of the interviews applied to the sample of the study, it was considered that in the Luis Rodríguez Valera Educational Institution there is very little ecological awareness, so it was necessary to build the proposal United we will take care of our house, the Earth that after applied and systematized their results. It was concluded that its execution was successful, achieving the formation of ecological awareness in both students and teachers.
\end{abstract}

Keywords: Strategies, environmental education, transversality, ecological awareness.

\title{
Resumo
}

A pesquisa denominada Estado da educação ambiental na Instituição educativa Luis Rodríguez Valera, no município de Valledupar, Cesar, procurou desenvolver diretrizes de educação ambiental na Institución Educativa Luis Rodríguez Valera, no município de Valledupar, Cesar. Teoricamente baseou-se nos postulados da Conferência do Tbilisi (1977), Declaração de Rio sobre Médio Ambiente e o Desenvolvimento (1993), Congresso Internacional Unesco PNAMU sobre Educação Formação e Formação Ambiental Moscou (1987), A Declaração de Comodoro Rivadavia.

Transformar Nosso Mundo: a Agenda 2030 para o Desenvolvimento Sustentável (Sauvé, 2014), além das Normativas jurídicas da Colômbia. Metodologicamente enquadrou-se no enfoque multimétodo e aplicada. Seu desenvolvimento se estruturou em seis trajetórias. A população esteve conformada por trezentos e oitenta e cinco (385) estudantes, um (1) reitor, dezenove (19) docentes. Com uma amostra não probabilística de noventa (90) estudantes, dez (10) docentes e um grupo focal de sete (7) estudantes; Com o análise dos resultados das entrevistas feitas à amostra do estudo se considerou que na Instituição Educativa Luis Rodríguez Valera há pouca consciência ecológica, por isso foi necessário construir a proposta Unidos cuidaremos de nossa casa, a Terra que depois de aplicada e sistematizados os resultados. Concluiu-se que a sua execução foi um sucesso, conseguido a transformação da consciência ecológica tanto nos estudantes como nos docentes.

Palavras-chave: Estratégias, educação ambiental, consciência ecológica. 


\section{Introducción}

A nivel mundial el hombre ha puesto en peligro su vida a causa de la manera como se relaciona con el medio ambiente, esta irresponsabilidad humana a propiciado gran desequilibrio e injusticias tanto sociales como ambientales debido a la apropiación, producción, consumo y el crecimiento explosivo poblacional, cuestiones que agravan la situación de la biosfera que está siendo degradada.

Ante esa situación surge la necesidad de preservar el ecosistema, por ello muchos ambientalistas han buscado la forma de transformar los materiales de uso cotidiano como botellas, envases de alimentos, entre otros originando nuevos productos o nuevas funciones con la finalidad de evitar daños al ecosistema, porque estos materiales duran muchos años para descomponerse; sin embargo, esto no es suficiente, es necesario sensibilizar a los individuos a que tengan una mirada sistémica y con su ayuda poder lograr el saneamiento ambiental y aproximarse de esa forma a la realidad geodinámica ambiental.

Por esa razón existe la urgencia de realizar actividades en todos los ámbitos enmarcadas desde la perspectiva ecologista conservacionista del medio, que promuevan una mirada integral del ambiente, donde las inadecuadas relaciones del hombre con la naturaleza y el desconocimiento de las leyes naturales han fragmentado el equilibrio del planeta, causando daños irreparables que afecta la vida de todos los seres que habitan en la tierra.

Los planteamientos anteriores han materializado la inclusión de la educación ambiental en el currículo, pero por una escasa formación que han tenido los docentes, la falta de visión integradora y global de la Educación Ambiental, ha traído como consecuencia que los estudiantes como sujetos educativos de cambio no estén integrando a la Educación Ambiental en sus prácticas ciudadanas desde una perspectiva socio-critica, transformadora de sí mismo y de su entorno en su praxis individual y colectiva de forma permanente.

Partiendo de lo emitido esta investigación se basó en desarrollar lineamientos de educación ambiental en la institución educativa Luis Rodríguez Valera, en el municipio de Valledupar - Cesar con el propósito de formar la conciencia ecológica conservacionista en cada uno de los actores y autores de la comunidad educativa. El trabajo se desarrolló en base a los criterios de enfoque multimétodos ejecutada en seis fases: la Fase I, titulada Contexto institucional, abordó la ubicación geográfica de la institución, reseña histórica, y situación ambiental institucional, problematización, objetivos, importancia $\mathrm{y}$ justificación, delimitación de la investigación.

La Fase II, llamada Marco teórico, vislumbró el estudio del arte de la educación ambiental, eventos realizados en diferentes países para afianzar la educación ambiental, estudios previos a nivel internacional, nacional, local, referentes teóricos conceptuales y la legislación ambiental de Colombia. La Fase III, titulada Abordaje metodológico, estudió la metodología de la investigación, población, muestra y técnicas e instrumentos. En la Fase IV denominada Resultados y discusión se expusieron los resultados de los instrumentos aplicados a los estudiantes, docentes, rector y grupo focal.

Seguidamente la Fase V titulada Lineamientos: Unidos cuidaremos de nuestra casa, La Tierra. En ella se desarrolló la justificación, objetivos, la sustentación teórica y la planificación del proceso de ejecución. En la Fase VI titulada Evaluación y sistematización, se desarrollaron las actividades, los logros generales, la evaluación de cada uno de los objetivos específicos, las conclusiones, bibliografías y anexos. 


\section{Importancia y justificación de la investigación}

La Educación Ambiental, ha venido postulándose con gran despliegue como un mecanismo pedagógico desde hace mucho tiempo, esta disposición en la educación retoma la enorme importancia que pueden tener las instituciones educativas para comprender la interacción que existe dentro de los ecosistemas, todo con el fin de entender el entorno y formar una cultura conservacionista donde el hombre aplique en todos sus procesos productivos, energías limpias (dando solución a los problemas ambientales), permitiendo de esta forma el desarrollo sostenible. En suma, la Educación Ambiental engloba un gran número de elementos como son: la naturaleza (medio ambiente) donde se definen los ecosistemas, la importancia de la atmósfera, el agua, el suelo, el flujo de materia y energía dentro de los diferentes entornos naturales, así como el comportamiento de las comunidades y poblaciones. (Plaza, 2017, p.3)

Por supuesto, también ayuda a conocer la interacción que hay entre el medio ambiente y el hombre cómo las actividades humanas influyen en los ecosistemas, cómo el ser humano ha aprovechado los recursos, así mismo brinda la descripción y consecuencias de la contaminación generada en las diferentes actividades, como se puede prevenir, que soluciones existen, promoviendo de una u otra forma el desarrollo sostenible y la conservación del entorno.

Por esas razones la importancia de educación ambiental está basada en la concienciación tanto de los niños, como adolescentes y adultos para conocer y respetar los elementos del medio, así como comprender sus relaciones dentro del mismo, adquirir un sentido crítico hacia lo que provocan sus acciones de degradación o desaparición de los elementos de los sistemas ecológicos o bien ayudar a resolver los problemas generados en los mismo, generar un sentido de pertenencia y responsabilidad con las acciones para aprender a minimizar su impacto en el medio que les rodea, para así ayudar a un desarrollo sostenible que garantice la subsistencia de las generaciones futuras.

Las situaciones expuestas, dejan ver que la educación ambiental desde sus normas, estrategias y actividades desde el Proyecto Educativo Institucional P.E.I y el currículo, pueden garantizar un verdadero cambio en el accionar y pensar de la comunidad generando la sensibilidad y conservación del medio ambiente.

La dimensión ambiental es de vital importancia en el momento de tener en cuenta la función de la enseñanza como sistema integral de procesos porque permite dinamizar la educación que busca el cambio de actitudes y convicciones, por lo que es relevante formar un individuo consciente del entorno en el que habita y su papel en la sociedad. Son estas razones válidas para analizar las prácticas pedagógicas y el currículo institucional, con el objetivo de cuestionar la educación ambiental hacia las comunidades educativas donde se puede contribuir con el desarrollo sostenible y cuidado del ambiente generando una sociedad más sensible y consciente de la significación que tiene el medio ambiente.

De la significación que tiene el estudio se justifica desde el punto de vista teórico, puesto que permitió analizar el modo de concebir y desarrollar EA al examinar las diferentes estructuras teóricas que le sirvieron de base a las afirmaciones que el autor de la investigación hace sobre los hechos estudiados en el ámbito de la educación ambiental.

Con relación a su aplicación, desde el punto de vista práctico, el estudio indicó posibles soluciones al problema planteado, quedando como muestra formal un estudio práctico del hecho investigado. Asimismo, benefició tanto a comunidad en donde se encuentra ubicada la institución educativa como al ambiente interno de la misma. 
En la perspectiva metodológica la investigación se justifica porque permitió diseñar los instrumentos de recolección de datos y posteriormente aplicarlos a la muestra del estudio, además con los datos obtenidos de manera cuantitativa se pudo ejecutar la discusión y análisis del estudio, que dieron respuestas al objetivo de la investigación, evaluar los resultados de aplicación de los lineamientos por el investigador para luego emitir las conclusiones.

En cuanto a su relevancia social, se justificó por la necesidad de la sociedad de enfrentar los retos que significan concienciar a la población desde una educación ambiental más real y efectiva para garantizar la preservación del planeta para las generaciones futuras prestando mayor atención a un accionar amigable y armonioso con el medio ambiente.

\section{Marco teórico}

Actualmente la educación enfrenta el reto de contribuir definitivamente con la solución de la problemática ambiental mundial. Por consiguiente, es necesario que este punto de vista, que desarrolle la Educación con amplios fines y objetivos de aprendizaje que puedan estar relacionados con la escuela y sus formas de educación. En tal sentido la Educación Ambiental es definida por la UNESCO-PNUMA (1990) como:

El proceso de reconocer valores y clarificar conceptos con el objeto de desarrollar habilidades y actitudes necesarias para comprender y apreciar las interrelaciones entre el hombre, su cultura y sus entornos biofísicas. La educación ambiental incluye también la práctica en la toma de decisiones y la autoformulación de un código de conducta sobre los problemas que se relacionan con la calidad ambiental. (p. 36)

Para García (como se citó en Sarango,
Sánchez y Landívar, 2016) la Educación ambiental es "el proceso de aprendizaje que debe facilitar la comprensión de las realidades del medioambiente, del proceso sociohistórico que ha conducido a su actual deterioro" (p. 185). Para Bedoy (como se citó en Martínez, 2010) el concepto de educación ambiental no es estático, evoluciona de forma paralela a como lo hace la idea de medio ambiente y la percepción que se tiene.

Los proyectos ambientales escolares tienen una proyección de carácter permanente y no son establecidos a corto ni mediano plazo, más bien su desarrollo educativo, pedagógicos $\mathrm{y}$ material son establecidos de manera indefinida hasta alcanzar su máximo desarrollado como proceso de aprendizaje en los estudiantes en procura generar en ellos actitudes de valoración y respeto por el medio ambiente. López (2011) expresó que los PRAE son proyectos pedagógicos que promueven el análisis y la comprensión de los problemas y las potencialidades ambientales locales, regionales y nacionales, y generan espacios de participación para implementar soluciones acordes con las dinámicas naturales y socioculturales.

La óptica de su quehacer es la formación desde una concepción de desarrollo sostenible, entendido como el aprovechamiento de los recursos en el presente, sin desmedro de su utilización por las generaciones futuras, con referentes espaciotemporales y sobre la base del respeto a la diversidad y a la autonomía y que contempla no sólo aspectos económicos sino sociales, culturales, políticos, éticos y estéticos en pro de una gestión sostenible del entorno (MEN, 2017).

Estos Proyectos propician en la escuela espacios para el desarrollo de estrategias de investigación y de intervención. Para Navarro (2007) las primeras, implican procesos pedagógico-didácticos e interdisciplinarios, cuyo 
fin es reflexionar críticamente sobre las formas de ver, razonar e interpretar el mundo y las maneras de relacionarse con él; igualmente, sobre los métodos de trabajo, las aproximaciones al conocimiento y, por consiguiente, la visión e interacción entre los diferentes componentes del ambiente. Las segundas, de intervención, implican acciones concretas de participación y de proyección comunitaria (Flores, 2015).

\section{Objetivo de la investigación}

Determinar el estado de la educación ambiental en la Institución Educativa Luis Rodríguez Valera, en el municipio de Valledupar, Cesar.

\section{Objetivos específicos}

Determinar la línea base de la Educación Ambiental en la Institución Educativa Luis Rodríguez Valera, en el municipio de Valledupar, Cesar.

Diseñar los lineamientos para fortalecer la Educación Ambiental en la Institución Educativa Luis Rodríguez Valera, en el municipio de Valledupar, Cesar.

Aplicar y evaluar la aplicación de las estrategias que fortalezcan la Educación Ambiental en la Institución Luis Rodríguez Valera, en el municipio de Valledupar, Cesar.

\section{Materiales y métodos}

\section{Localización del estudio}

La Institución Educativa Luis Rodríguez Valera se encuentra ubicada en el corregimiento de Guaimaral, al sur de la ciudad de Valledupar, Cesar.

\section{Metodología de investigación}

El presente estudio pretende determinar el estado de la Educación Ambiental en la
Institución Educativa Luis Rodríguez Valera, en el municipio de Valledupar, Cesar, o la percepción e imaginarios que sobre ella se tiene en el ámbito comunitario, hecho por lo cual se considera que es una investigación que parte del enfoque histórico hermenéutico. Se trata de una investigación cualitativa en donde se genera la búsqueda de una descripción de una realidad en torno a la dimensión ambiental, además de ser cuantitativa, debido a que se analizan datos y porcentajes sobre encuestas a la población y su percepción frente al objeto de estudio, para de esta manera y a partir de un grupo de resultados realizar ajustes a nivel educativo y formativo, para generar cambios en la forma de actuar y pensar en la población estudiantil. Para así tratar de lograr la formación de la conciencia ecológica a través de la Educación Ambiental. Es por ello que es un proyecto de investigación de Desarrollo Social Humano.

\section{Población y muestra}

Las personas que participaron en la investigación fueron la comunidad estudiantil, docentes y el rector de la Institución Educativa Luis Rodríguez Valera con 385 estudiantes, un rector, y diecinueve (19) docentes. Específicamente para la investigación la muestra estuvo conformada por noventa (90) estudiantes de primero al noveno grado, un rector, diez (10) docentes y siete (7) estudiantes del grupo focal, que fueron tres (3) estudiantes de educación primaria y cuatro (4) de secundaria.

\section{Técnicas e instrumentos}

Se aplicó una encuesta a 10 docentes con el objetivo de generar un primer acercamiento al tema de investigación. De igual forma se aplicaron entrevistas semiestructuradas, a los estudiantes del primero a1 noveno grado, con una serie de preguntas de carácter abierto, así mismo al rector se le hizo una entrevista abierta a profundidad con la finalidad de ver si los docentes en sus praxis realizan proyectos ambientales y la relacionan con todas las disciplinas del saber. 


\section{Resultados y discusión}

La presentación y análisis de los resultados que se realizaron en el estudio tuvo como fin primordial Determinar el estado de la Educación Ambiental en la Institución Educativa Luis Rodríguez Valera, en el municipio de Valledupar, Cesar. Esta fase se ejecutó a través del desarrollo de los objetivos específicos de la investigación, por emplear la metodología aplicada y teniendo un enfoque multimétodo, se construyeron un conjunto de gráficos porcentuales de acuerdo con los criterios de cada ítem los cuales reflejaron los resultados obtenidos. A estos resultados se les aplicó la estadística descriptiva. El Gráfico 1 mostró los resultados a la pregunta ¿Conocen el proyecto del medio ambiente que se realiza en la institución educativa? El resultado mostró que el $72 \%$ la población estudiantil encuestada dijo que No lo conocen, un $13 \%$ manifestaron Sí lo conocen, el $15 \%$ comenta que solo saben de las actividades que se realizan y ningún otro aspecto del proyecto. Estos resultados demuestran un desconocimiento general del proyecto ambiental que opera en la institución. Los resultados obtenidos están en disparidad al estudio realizado por González (2012) cuando expresó la importancia de implementar una práctica educativa orientada a

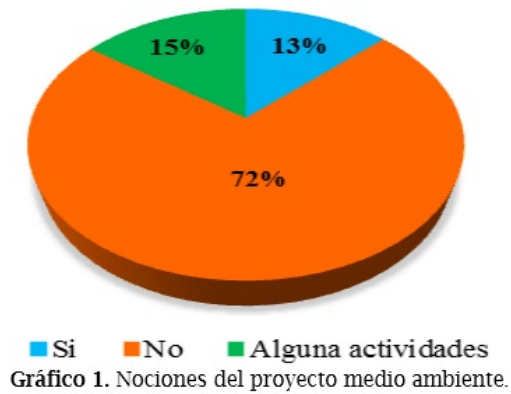

desarrollar una profunda conciencia ecológica que reconozca la interdependencia de todos los seres humanos con sus semejantes y con las demás especies, aún más, con los fenómenos planetarios que día a día se manifiestan como individualidades y sociedad.
De igual forma difiere con lo emanado por la Conferencia de Tbilisi (1977), en la cual se anuncia que la Educación Ambiental deberá estar orientada hacia la resolución de los problemas ambientales, formando a los estudiantes una conciencia ambientalista y, por consiguiente, participen de manera activa en el cuidado del ambiente. Con base a lo anterior, es necesario encauzar los sistemas educativos con mayor idoneidad, realismo y una mayor interpenetración del medio natural y social, con miras a facilitar el bienestar de las comunidades humanas y del entorno natural.

En el Gráfico 2 se muestran los resultados de la entrevista realizada a los docentes y respondió a la interrogante ¿Por qué a los estudiantes les falta cultura ambiental? El $60 \%$ de los docentes manifestaron que la falta de cultura ambiental se debe a que los estudiantes no han recibido una Educación Ambiental adecuada; el $24 \%$ de los docentes cree que les falta motivación; el $8 \%$ de los docentes manifestaron que los estudiantes no tienen costumbres familiares por el cuidado del medio ambiente. Por último, el $8 \%$ expresaron que los estudiantes No tienen interés en recibir información.

Estos resultados difieren a las conclusiones de la investigación de Rivas (2006) cuando alegó que la dotación de áreas verdes en espacios educativos permitirá a los actores y autores del proceso educativo establecer acuerdoscon el ambiente, aprovechando los espacios para realizar actividades recreativas, de disfrute y descanso, proporcionando la formación de una cultura conservacionista, protectora, donde el

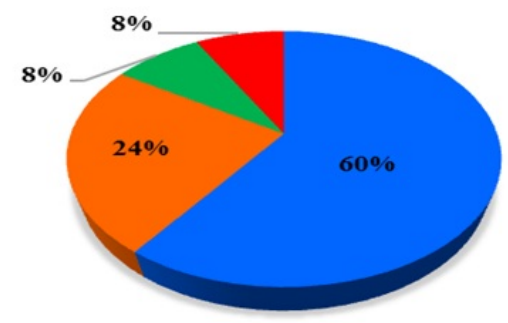

E Falta de educación ambiental $=$ Falta de motivación Gráfico 2. ¿Por qué a los estudiantes les falta cultura ambiental? 
aprovechamiento del entorno se realice con una conciencia sistémica.

De igual manera dista a lo emitido por el Ministerio de Educación Nacional y Ambiente (2014) cuando expresó que la Educación Ambiental es un proceso educativo, integral e interdisciplinario que enseña a contemplar el ambiente como un todo, involucrando a toda la población en la identificación y resolución de problemas, utilizando las herramientas del conocimiento, valores, actitudes y habilidades, para la toma de decisiones y la participación activa y organizada del medio ambiente. La entrevista a profundidad realizada al Rector se presenta en el Tabla 1.

\begin{tabular}{|c|c|c|}
\hline LN & Contenido de la entrevista & Categoria \\
\hline 1 & Buezas dias Doctof con permiso, como esta. Le agradezco la opertunidad que me brinda y su & \\
\hline 2 & colaboración para responder a mis preguntas, son parte mi imestigación de maestria. Iniciaria & PRAE \\
\hline 3 & esta eatrevista preguntandole: ¿Como se conciben los PRAE en la institución? R. Bien. los & \\
\hline 4 & propecto se aplican para comprender y reflexionar sobre la comprension de los problemas & \\
\hline 5 & ambientales que se observan en ta institucion, creo que si algo hace falta es visualizar esos & \\
\hline 6 & mismos problemas nivel regional y nacional Además, podria expresar gue los PRAE se & \\
\hline 7 & desarrollan de manera ocastonal y de acuerdo a las efemérides o ante algün fenómeno natural, & PRAE, Planes de estudio \\
\hline 8 & por lo tanto hay que trabajar más en la constancia de su aplicabilidad en la instirución. Los & \\
\hline 9 & Planes de estudio que se ejecutan ea la institudión desarrollan competencias ambientalista & \\
\hline 10 & estudiantes? $\mathrm{R}$, aunque es d deber ser de los PRAE, creo que esa parte está debilitada, en los & \\
\hline 11 & planes de esfudio si se desarrollan la educación ambiental, pero se afianzan más, o sea & Transversalidad \\
\hline 12 & atienden las diversas areas academicas en su fin cognitivo, pero no se observan las & Conceptuales, Procedimentales \\
\hline 13 & competencias ambientalistas como parte integral de un proceso transversal, lo que hace que & Axdológicos \\
\hline 14 & los estudiantes desarrollen solo las competerias conceptuales que no se llevan a la praictica & \\
\hline 15 & y mucho menos se insertan los valores como parte esencial de la vida, por la carencia de & \\
\hline 16 & aplicablididad y de la inserción de peoyectos ambientales en todas las áreas del saber. & \\
\hline 17 & Entonces hay que conocer la aplicabilidad de la Ley 99 que hace referencia a los & Ley 99 de 1993 \\
\hline 18 & programas de educacióa ambiental? $\mathrm{R}$. Si, claro y por ello te he abierto las puertas de la & \\
\hline 19 & institución para que nos ayude a desarrollar programas de educación ambiental, donde los & Transversalidad \\
\hline 20 & docentes puedan ejecutar en sus praxis proyectos ambientales insertándolos en la & \\
\hline 21 & rransversalidad, se que necesitamos procesos de formacion y actualización y ante ello acudo & \\
\hline 22 & a ti, para que nos ayude a promover y desarrollar la participacion comunitaria en actividades & Desarrollo sostenible \\
\hline 23 & y programas de protección ambiental, con el desarrollo sastenible y de manejo adecuado de & \\
\hline 24 & los recursos naturales renosables. ¿En la institución educativa se implementa la ensen̂anza & \\
\hline 25 & obligatoria de la protección del ambiente? R. En estos momentos no, de verdad que durante & \\
\hline 26 & el ano escolar no se implementó como lo señala la Ley IIS al decir que en la enserianza debe & Ley 115 de 1993 \\
\hline 27 & ser abligatoria de la proteccion del amblente, la ecologia y la preservacioin de los recursos & \\
\hline 28 & narurales. Esa es una parte que hay que fortalecer en lá institución, quizas por ello los & \\
\hline 29 & estudiantes no hayan adquirido una conciencia para la conservadón protección y & \\
\hline 30 & mejoramiento del medio ambiente. Pero, vamos a ayudamos mutuamente, la institución te & \\
\hline 31 & servirá de gula para el desarrollo de tu proyecto de grado y tú nos ayuda a implementar los & Ley 115 de 1993 \\
\hline 32 & projectos de educación ambiental en el colegio juguemos a ganar-ganar y de esta manera la & Enseñanza obliqatoria \\
\hline 33 & implementación obligatoria de educación ambienfal no se verá como una camisa de fuerza & \\
\hline 34 & sino como una necesidad institucional, humanista, social, educativa del medio amblente. & \\
\hline 35 & Donde los docentes, estudiantes, representantes y comunidad en general se apropien de los & \\
\hline 36 & conocimientos, saberes y formas de aprowimarse individual y colectivamente, a un manejo & Manejo sostenible \\
\hline 37 & sostenible de las realidades ambientales, a través de la generación de un marco ético, que & Conceptuales, Proxedimentales \\
\hline 38 & enfatice en actirudes de valaracion y repeto por el ambiente. ¿Usted como gerente desarrolla & Adologicos \\
\hline 39 & actividades para el manejo eficiente de los recursos naturales? $\mathrm{R}$. Con mucha pena tengo & \\
\hline 40 & que decirte que no, basta ahora no be propiciado el desarrollo de actividades que conilleven & \\
\hline 41 & al manejo eficiente de los recursos naturales a pesar que su preservación es obligación de & Ley 99 de 1993 \\
\hline 42 & todos y en mi condición de rector debo tenerlo presente, porque los nathos son el futuro del & \\
\hline 43 & pais y si ellos adquieren conciencia en el caidado y presenvación del ambiente, el planeta & \\
\hline 44 & continuará vivo y nosotros en el. 1.0 que quiero decir es que si se necesita desarrollar propwctos & Enseñanza obligatoria \\
\hline 45 & de edicacion ambiental pura lograr la preservaciont, restauracioin, consenvacion, mejoramicnto & Ley 115 de 1994: \\
\hline 46 & y utîización racional de los recuessas nafurales renovables, especialmente en el agua, la fauma & \\
\hline 47 & y fora, además que nuestros estudiantes sean número 1 en el desarrollo armónico de los & Decreto 2811 de 1974 \\
\hline 48 & recursos naturales para beneficio de la salud y el bienestar de todos los babitantes. Muchas & \\
\hline 49 & gracias doctor por su receptividad y espontaneidad en la realizacion de la entrevista. Buen & \\
\hline 50 & dia. & \\
\hline
\end{tabular}

Tabla 1. Protocolo de la Entrevista a profundidad. Siste. por Rivera, J. (2009). 
Educación Ambiental en la Institución Educativa Luis Rodríguez Valera, en el municipio de Valledupar, Cesar

Propuesta. Unidos cuidaremos de nuestra casa, La tierra.

\begin{tabular}{|c|c|c|c|c|c|c|}
\hline $\begin{array}{r}\text { Obje } \\
\text { cui }\end{array}$ & $\begin{array}{l}\text { vo general: } \\
\text { aremos de nu }\end{array}$ & $\begin{array}{l}\text { oncienciar la } \\
\text { stra casa, la } t\end{array}$ & $\begin{array}{l}\text { aportancia de } \\
\text { erra Institución }\end{array}$ & $\begin{array}{c}\text { conservar el a } \\
\text { Educativa } L\end{array}$ & $\begin{array}{l}\text { biente porque } \\
\text { s Rodríguez V }\end{array}$ & $\begin{array}{l}\text { nidos } \\
\text { era. }\end{array}$ \\
\hline $\begin{array}{l}\text { Objetivos } \\
\text { Específicos }\end{array}$ & Estrategia & Actividades & Responsables & Recursos & Logros & $\begin{array}{l}\text { Medios de } \\
\text { Verificación }\end{array}$ \\
\hline Sensibilizar & Formación & Selección de & Docente & Selección por & los & Actuación de \\
\hline la & de grupos & los grupos & investigador & aula de un & grupos focales, & los grupos \\
\hline comunidad & focales $\quad y$ & focales. & & estudiante. & miembros & focales. \\
\hline educativa & Charla & Intervención & & Materiales & de la Junta & Asistencia de \\
\hline sobre los & Ambientalista & de los grupos & & de papelería. & Comunal, & los invitados \\
\hline problemas & & focales. & & Computador & & a la charla \\
\hline ambientales & & Invitación & & Video beam & Consejo & sobre \\
\hline que hay en & & a la Junta & & Refrigerio & Comunitario & importancia \\
\hline la I.E. Luis & & Comunal, & & & y Padres & del ambiente \\
\hline Rodríguez & & Consejo & & & representantes & en la vida del \\
\hline Valera & & Comunitario & & & de la & hombre. \\
\hline & & y Padres y & & & institución & \\
\hline & & representantes & & & se sensibilicen & \\
\hline & & de la & & & sobre los & \\
\hline & & institución & & & problemas & \\
\hline & & para darles & & & ambientales & \\
\hline & & a conocer los & & & & \\
\hline & & problemas & & & & \\
\hline & & ambientales & & & & \\
\hline & & de la & & & & \\
\hline & & institución. & & & & \\
\hline & & Organizar & & & & \\
\hline & & charla & & & & \\
\hline & & sobre & & & & \\
\hline & & importancia & & & & \\
\hline & & del ambiente & & & & \\
\hline & & en la vida del & & & & \\
\hline & & hombre. & & & & \\
\hline Participar & Campaña de & Invitación & Docente & Artículos & Que & Con \\
\hline & limpieza en la & los & investigador & de limpieza. & estudiantes, & escuela limpia \\
\hline jornada de & institución & estudiantes, & Estudiantes & Hidratación. & padres & libre de \\
\hline limpieza en & & padres & Padres & & representantes & escombros. \\
\hline la I.E. Luis & & representantes & representantes & & y miembros & \\
\hline Rodríguez & & y miembros & Junta & & activos de la & \\
\hline Valera & & activos de la & Comunal & & comunidad & \\
\hline & & comunidad a & Consejo & & participen & \\
\hline & & participar en & Comunal & & la jornada de & \\
\hline & & la campaña & & & recogida & \\
\hline & & de limpieza de & & & escombros $\quad y$ & \\
\hline & & $\begin{array}{l}\text { la institución } \\
\text { educativa. }\end{array}$ & & & $\begin{array}{l}\text { limpieza de la } \\
\text { escuela }\end{array}$ & \\
\hline
\end{tabular}




\begin{tabular}{|c|c|c|c|c|c|c|}
\hline & & $\begin{array}{l}\text { Recogiendo los } \\
\text { escombros en } \\
\text { la institución. } \\
\text { Limpieza de la } \\
\text { escuela. }\end{array}$ & & & & \\
\hline $\begin{array}{l}\text { Conocer la } \\
\text { importancia } \\
\text { de cuidar } \\
\text { la tierra } \\
\text { porque es } \\
\text { nuestra } \\
\text { casa en la } \\
\text { I.E. Luis } \\
\text { Rodríguez } \\
\text { Valera }\end{array}$ & $\begin{array}{l}\text { Taller: } \\
\text { Unidos } \\
\text { cuidaremos } \\
\text { de nuestra } \\
\text { casa, la tierra }\end{array}$ & $\begin{array}{l}\text { Invitación a } \\
\text { los estudiantes } \\
\text { Expectativas } \\
\text { del taller } \\
\text { Unidos } \\
\text { cuidaremos } \\
\text { nuestra casa, } \\
\text { la tierra. } \\
\text { Desarrollo de } \\
\text { la temática. } \\
\text { Conclusiones. } \\
\text { Retroalimentaciór }\end{array}$ & $\begin{array}{l}\text { Docente } \\
\text { investigador }\end{array}$ & $\begin{array}{l}\text { Materiales } \\
\text { de papelería. } \\
\text { Computador } \\
\text { Video beam }\end{array}$ & $\begin{array}{l}\text { Que los } \\
\text { estudiantes } \\
\text { reconozcan que } \\
\text { la tierra es } \\
\text { nuestra casa y } \\
\text { que debemos } \\
\text { cuidarla. }\end{array}$ & $\begin{array}{l}\text { Imágenes } \\
\text { fotografiadas. } \\
\text { Lista de } \\
\text { asistencia. } \\
\text { Afiches, } \\
\text { Papelotes de } \\
\text { trabajo }\end{array}$ \\
\hline
\end{tabular}

Tabla 2. Plan de acción. Estudiantes. Siste. por Rivera, J. (2008)

\begin{tabular}{|c|c|c|c|c|c|c|}
\hline \multicolumn{7}{|c|}{$\begin{array}{l}\text { Objetivo general: Concienciar la importancia de conservar el ambiente porque unidos } \\
\text { cuidaremos de nuestra casa, la tierra Institución Educativa Luis Rodríguez Valera. }\end{array}$} \\
\hline $\begin{array}{l}\text { Objetivos } \\
\text { Específicos }\end{array}$ & Estrategia & Actividades & Responsables & Recursos & Logros & $\begin{array}{l}\text { Medios de } \\
\text { Verificación }\end{array}$ \\
\hline $\begin{array}{l}\text { Conocer la } \\
\text { importancia } \\
\text { de cuidar } \\
\text { la tierra } \\
\text { porque es } \\
\text { nuestra } \\
\text { casa en la } \\
\text { I.E. Luis } \\
\text { Rodríguez } \\
\text { Valera }\end{array}$ & $\begin{array}{l}\text { Taller: } \\
\text { Unidos } \\
\text { cuidaremos } \\
\text { de nuestra } \\
\text { casa, la } \\
\text { tierra. }\end{array}$ & \begin{tabular}{l}
\multicolumn{1}{c}{ Invitación a } \\
los docentes para \\
participar en un \\
taller titulado Unidos \\
cuidaremos de \\
nuestra casa, la \\
tierra. \\
$\checkmark \quad$ Expectativas \\
del taller Unidos \\
cuidaremos nuestra \\
casa, la tierra. \\
$\checkmark$ Desarrollo de la \\
temática. \\
$\checkmark$ Conclusiones. \\
$\checkmark$ Retroalimentación.
\end{tabular} & $\begin{array}{l}\text { Docente } \\
\text { investigador }\end{array}$ & $\begin{array}{l}\text { Materiales } \\
\text { de papelería. } \\
\text { Computador } \\
\text { Video Beam } \\
\text { Refrigerio }\end{array}$ & $\begin{array}{l}\text { Que los } \\
\text { docentes } \\
\text { conciencien } \\
\text { reconozcan } \\
\text { que la tierra } \\
\text { es nuestra } \\
\text { casa y que } \\
\text { debemos } \\
\text { cuidarla. }\end{array}$ & $\begin{array}{l}\text { Fotografías. } \\
\text { Lista de } \\
\text { asistencia. } \\
\text { Afiches, } \\
\text { Papelotes } \\
\text { de trabajo }\end{array}$ \\
\hline $\begin{array}{l}\text { Reconocer } \\
\text { la } \\
\text { importancia } \\
\text { de las } 5 \text { R }\end{array}$ & $\begin{array}{l}\text { Taller: Con } \\
\text { las } 5 \mathrm{R}\end{array}$ & $\begin{array}{l}\checkmark \quad \text { Invitación a } \\
\text { los docentes para } \\
\text { participar en un taller } \\
\text { titulado: Con las } 5 \mathrm{R}\end{array}$ & $\begin{array}{l}\text { Docente } \\
\text { investigador }\end{array}$ & $\begin{array}{l}\text { Materiales } \\
\text { de papeleria } \\
\text { y reciclaje } \\
\text { Computador }\end{array}$ & $\begin{array}{l}\text { Que los } \\
\text { estudiantes } \\
\text { reconozcan } \\
\text { la }\end{array}$ & $\begin{array}{l}\text { Imágenes } \\
\text { fotografiadas. } \\
\text { Lista de } \\
\text { asistencia. }\end{array}$ \\
\hline
\end{tabular}




\begin{tabular}{|c|c|c|c|c|c|c|}
\hline $\begin{array}{l}\text { para } \\
\text { cuidar el } \\
\text { planeta en } \\
\text { la I.E. Luis } \\
\text { Rodríguez } \\
\text { Valera }\end{array}$ & planeta. & $\begin{array}{l}\text { cuidaremos nuestro } \\
\text { planeta. } \\
\checkmark \text { Expectativas del } \\
\text { taller: Con las } 5 \\
\text { R cuidaremos nuestro } \\
\text { planeta. } \\
\checkmark \text { Desarrollo de la } \\
\text { temática. } \\
\checkmark \text { Conclusiones. } \\
\checkmark \text { Retroalimentación. }\end{array}$ & & Video beam & $\begin{array}{l}\text { importancia } \\
\text { de las } 5 \mathrm{R} \\
\text { para cuidar } \\
\text { el planeta }\end{array}$ & $\begin{array}{l}\text { Afiches, } \\
\text { Papelotes } \\
\text { de trabajo }\end{array}$ \\
\hline $\begin{array}{l}\text { Manejar la } \\
\text { transversa- } \\
\text { lidad de la } \\
\text { educación } \\
\text { ambiental } \\
\text { en las } \\
\text { áreas del } \\
\text { curriculo }\end{array}$ & $\begin{array}{l}\text { Taller: } \\
\text { transversa- } \\
\text { lidad del eje } \\
\text { ambiente en } \\
\text { el pensum } \\
\text { de estudio }\end{array}$ & $\begin{array}{l}\checkmark \quad \text { Lectura reflexiva } \\
\text { del programa PRAE y } \\
\text { su aplicabilidad en la } \\
\text { escuela. } \\
\checkmark \quad \text { Visualización } \\
\text { de actividades a } \\
\text { través de videos del } \\
\text { YouTube referentes al } \\
\text { ambiente. } \\
\checkmark \quad \text { Insertar } \\
\text { Educación Ambiental } \\
\text { como eje vertical del } \\
\text { pensum de estudio. } \\
\checkmark \text { Reflexiones finales. }\end{array}$ & $\begin{array}{l}\text { Docentes } \\
\text { investigador }\end{array}$ & $\begin{array}{l}\text { Computador } \\
\text { Video beam }\end{array}$ & $\begin{array}{l}\text { Aplicación } \\
\text { del PRAE } \\
\text { en el } \\
\text { proceso de } \\
\text { enseñanza y } \\
\text { aprendizaje } \\
\text { en todas las } \\
\text { asignaturas. }\end{array}$ & $\begin{array}{l}\text { Aplicación } \\
\text { de las } \\
\text { temáticas } \\
\text { de } \\
\text { Educación } \\
\text { Ambiental } \\
\text { en todas las } \\
\text { disciplinas } \\
\text { del } \\
\text { curriculo } \\
\text { escolar. }\end{array}$ \\
\hline
\end{tabular}

Tabla 3. Plan de Acción. Docentes. Siste. por Rivera, J. (2018).

$\mathrm{Su}$

\section{Evaluación y sistematización de la propuesta}

1. Objetivo específico: Sensibilizar a la comunidad educativa sobre los problemas ambientales que hay en la I.E. Luis Rodríguez Valera.

2. Estrategia: Formación de grupos focales y charla ambientalista.

3. Actividad: Organizar una charla sobre la importancia del ambiente en la vida del hombre.

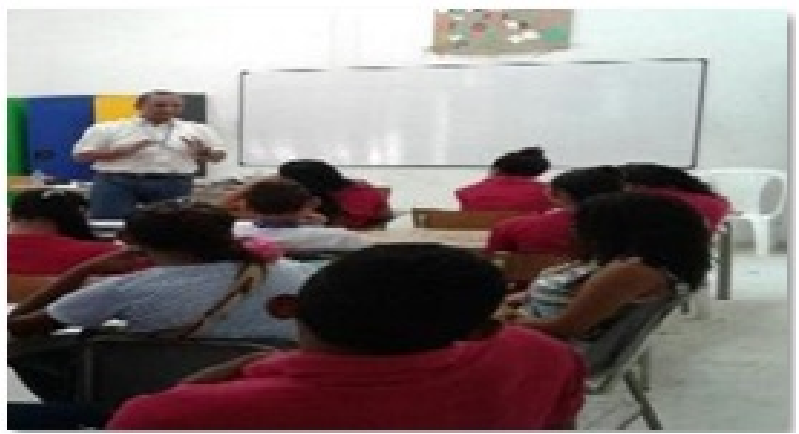

Imagen 1. Charla importancia del ambiente en la vida del hombre. Fuente: Archivo personal.

La Imagen 1 muestra la ejecución de la charla dirigida a los padres y representantes de la institución, a la Junta Comunal, y al Consejo 
Comunitario, en el cual se explicó la importancia de tener un ambiente acorde, respetando el medio ambiente para lograr una vida sana, de igual manera se explicó el desarrollo del proyecto y la importancia de su participación para la ejecución de este.

Fue una jornada exitosa en la parte de sensibilización y aceptación del proyecto. Los participantes reflexionaron sobre las condiciones ambientales de su sector, emitiendo la necesidad de resolver el problema ambiental dentro y fuera de la escuela. Esta reacción de la comunidad educativa permitió la elaboración de planes de acción para lograr solventar los problemas ambientales del entorno de la I.E. Luis Rodríguez Valera, descubriendo la importancia de cuidar el ambiente debido a que el ecosistema es nuestro hogar. Esto conllevó a que todos los actores se involucraran en gestionar los problemas ambientales que existen en la institución educativa y en la comunidad, participando en las actividades propuestas por el docente investigador.

Es pertinente expresar que los encuentros fueron exitosos, pues se logró la intervención de todos a favor de la educación ambiental. Lo anterior marca un nuevo rumbo a esta institución, que ha ido tomando conciencia de lo importante de la educación ambiental para lograr una mejor calidad de vida. Logro que parte de lo propio y de las inmensas riquezas que poseen las comunidades rurales, reconociendo la necesidad de líderes que motíven las gestiones ambientales en la búsqueda de mejorar y transformar la educación ambiental. Un proceso que está basado en la interdisciplinariedad y transversalidad, haciendo partícipe a la comunidad educativa en general de las actividades encaminadas hacia el conocimiento, acciones de cambio y conciencia sobre la educación ambiental.

1. Objetivo específico: Incluir las temáticas ambientales como eje transversal en las áreas del currículo.

2. Estrategia: Taller: Transversalidad del eje ambiente en el pénsum de estudio.

3. Actividad: Desarrollo de la temática.

\begin{tabular}{|c|c|c|c|}
\hline Áreas & Contenidos & Indicador de logros & Actividades \\
\hline Ciencias & Medio & $\begin{array}{lll}\text { Integra } & \text { los } & \text { saberes } \\
\end{array}$ & https://www.youtube.com/watch?v= \\
\hline \multirow[t]{11}{*}{ Naturales } & ambiente & /conocimientos $\quad \mathrm{y}$ & ZiSboskv0HA https://www.google.com \\
\hline & y problemas & experiencias & $\overline{\text { url? sa }=\text { t\&r ct }=\mathrm{j} \& q=\& \text { esrc }=\text { s\&source }}$ \\
\hline & ambientales. & actuar individual $\mathrm{y}$ & $=$ web\&cd $=5 \&$ cad $=$ rja\&uact $=8 \&$ ved $=$ \\
\hline & & colectivamente. & 0ahUKEwiK4o K8ybHUAhWJzz4KHd \\
\hline & & & w- AO4QFgg9MAQ\&url=https $\% 3 \mathrm{~A} \% 2$ \\
\hline & & & $\overline{\mathrm{F} \%}$ 2Feloceanografo.wikispaces.com\%2 \\
\hline & & & $\overline{\text { Ffile } \% 2 \text { Fview } \% 2 \text { FEcolog } \% 25 \mathrm{C} 3 \% 25 \mathrm{AD}}$ \\
\hline & & & a $\% 2 \mathrm{C} \% 2 \mathrm{BM}$ edio $\% 2 \mathrm{BAmbiente} \% 2 \mathrm{By} \% 2$ \\
\hline & & & B Des.\%2BSostenible-Iv\%25C3\%25A1n. \\
\hline & & & ppt\&usg=AFQjCNF mkEk5rm9QphNJ \\
\hline & & & 2xXkgxhsQTIR5w \\
\hline \multirow{4}{*}{$\begin{array}{l}\text { Ciencias } \\
\text { Sociales }\end{array}$} & Importancia & activamente & http://www.esap.edu.co/portal/downlo \\
\hline & de las & y se interesa & ad/plan_ambiental/7.Cartilla-de-Educa \\
\hline & conductas & responder a & cion-Ambiental.pdf https://www.youtu \\
\hline & $\begin{array}{l}\text { ambientales } \\
\text { sostenibles }\end{array}$ & $\begin{array}{l}\text { necesidades de } \\
\text { entorno. }\end{array}$ & be.com/watch?v=ymr7l5q8b-8 \\
\hline
\end{tabular}


Educación física

$\begin{array}{ll}\text { Educación } & \text { Fenómenos } \\ \text { religiosa } & \text { naturales. }\end{array}$

$\begin{aligned} & \text { Ética } \\ & \text { valores }\end{aligned}$
Educación
artística

Lengua
castellana

Inglés

Matemáticas Matemática ecológica. Situaciones problemáticas. física y salud

Elaboración de juegos con recursos del medio.

Medios masivos folletos, carteles, soportes gráficos. naturaleza.
Trabaja contenidos de tradicionales comunicación:

Vocabulario Emplea información del medio nueva y la interpreta en ambiente y su contexto. Asocia los conceptos abordados en la construcción de ideas Adopta comportamiento en la vida cotidiana acorde con la postura de defensa y Recuperación del equilibrio ecológico, social y cultural. el nivel local como global, y participo en iniciativas a su favor.

https://www.youtube.com/watch?v= H18Q4XfB1P0

https://www.youtube.com/watch?v= EIXzcXZ1f9M

https://es.slideshare.net/unir2010/de

porte-y-medio-ambiente-local

https://www.youtube.com/watch?v= D0NXzvLoKWc

https://www.youtube.com/watch?v= L-SAy2FA6bw

https://es.slideshare.net/riveraleos/eti ca-valores-y-medio-ambiente.

https://www.youtube.com/watch?v= sQi7owiYUUw un

Adopta

as

un https://www.youtube.com/watch?v= rBfrqpN6BZ4

https://www.youtube.com/watch?v= Cv3WJyCW-CQ

https://www.youtube.com/watch?v= ciecv6Cra6c

https://www.youtube.com/watch?v= ostCEliuDA4

https://www.youtube.com/watch?v= OHJdAbRucPE

https://www.youtube.com/watch?v= $\underline{\text { SO769aZdUcM }}$

https://www.youtube.com/watch?v= 2D62D1pzxrY

https://www.youtube.com/watch?v= 2Iy92z6WOqI

https://www.youtube.com/watch?v= bSJ1jpP0hok

https://www.youtube.com/watch?v= QKrOVIDUMJQ 


\begin{tabular}{|c|c|c|c|}
\hline \multirow[t]{10}{*}{ Tecnología } & Visitas a & Valora las actitudes & https://www.youtube.com/watch?v= \\
\hline & webquest & de los estudiantes & $\overline{\mathrm{jJ}} 21 \mathrm{WgB} 1 \mathrm{dhs}$ \\
\hline & sobre temas & hacia la tecnología, su & \\
\hline & ambientales. & sensibilización social $\mathrm{y}$ & \\
\hline & & ambiental, curiosidad, & \\
\hline & & cooperación y trabajo & \\
\hline & & en equipo, apertura & \\
\hline & & intelectual, búsqueda & \\
\hline & & y manejo de la in- & \\
\hline & & $\begin{array}{l}\text { formación, y deseo de } \\
\text { informarse. }\end{array}$ & \\
\hline
\end{tabular}

Tabla 4. Transversalidad del eje ambiente en el pensum de estudio. Inclusión de Educación Ambiental en el pénsum de estudio. Fuente: PRAE (2016). Siste. por Rivera, J. (2018).

\section{Conclusiones}

Una vez finalizada la investigación Educación Ambiental en la institución educativa Luis Rodríguez Valera, en el municipio de Valledupar - Cesar, se procedió a elaborar las conclusiones, las cuales tuvieron como referentes los objetivos del estudio, se estimó pertinente la emisión de consideraciones, expuestas de forma resumida capaz de recoger las ideas centrales de los resultados y de la aplicación de los lineamientos. En consecuencia, se formulan las siguientes conclusiones.

$\mathrm{Al}$ abordar la línea base de la Educación Ambiental en la Institución Educativa Luis Rodríguez Valera, en el municipio de Valledupar - Cesar, se obtuvo que los estudiantes desconocen la terminología asignadas al medio ambiente, además la aplicación de los PRAE no impactaron en la población estudiantil, los docentes se encuentran desmotivados, ocasionando que no visualicen la importancia de la Educación Ambiental dentro del pensum de estudio, creyendo que solo le resta tiempo para cumplir con el pensum de estudio. En referencia a la aplicación de la transversalidad, se evidenció desconocimiento en la inserción del área ambiente como eje central.

Lo que conllevó a diseñar una estrategia que permitiera a los docentes ejecutar su praxis educativa insertando el área ambiente en todas las asignaturas con actividades fáciles y pedagógicas, las cuales permitieron la motivación de los estudiantes y docentes para realizar actividades en todas las asignaturas sobre el medio ambiente con el fin de activar los valores de compromiso, responsabilidad, corresponsabilidad, formación de una conciencia ecológica, participación, y con ello, crear áreas verdes, ubicar la basura en un lugar idóneo dentro de la IE Luis Rodríguez Valera, por ser importante para la salud colectiva, el embellecimiento de la organización educativa, además de reconocer la importancia de que tiene el planeta por ser el hogar en su cuidado y preservación, logrando un ambiente más agradable, sano, con un desarrollo sostenible y sustentable.

La aplicación y evaluación de las estrategias utilizadas en los lineamientos permitió fortalecer la educación ambiental en la institución Luis Rodríguez Valera, incentivó a los docentes, estudiantes y comunidad a buscar un ambiente sano, donde la belleza de la naturaleza sea el reflejo del hombre, además del propiciar espacios de capacitación donde los participantes adquirieran las competencias cognitivas para cuidar y preservar el ambiente, así mismo se logró ambientes encantadores como los trabajados en el 
nicho ecológico, la casita ecológica y los jardines que por grado utilizaron en su construcción materiales con larga vida para desintegrarse como el caucho, el plástico, destacando las jardineras y decoraciones con cucharas desechables.

Otro logro relevante fue el afianzamiento de las relaciones entre el personal docente y los estudiantes con acciones amigables con el medio ambiente, además la participación de los diferentes sectores de la comunidad educativa desarrollando sus capacidades sociales para el diálogo y la toma de decisiones, lo que implica un proceso continuo de mejora para impulsar métodos en la búsqueda de alternativas de participación y compromiso ambiental.

Así mismo, se reflexionó sobre la práctica para transformar el currículo y sus formas de hacer, organizarse y relacionarse, teniendo como referencia la Educación Ambiental y el desarrollo sostenible.

\section{Recomendaciones}

Una vez finalizada la investigación se recomienda:

Al Rector, generar procesos de formación y apropiación ambiental dentro de la comunidad educativa, para lograr el desarrollo de una cultura y conciencia ambiental. Vigilar la aplicabilidad de los proyectos en los cuales deben estar presente la preservación del ambiente.

A los docentes, aplicar las estrategias presentadas en los lineamientos para afianzar en sus clases el compromiso y responsabilidad de los estudiantes en el cuidado del ambiente, además de estar en continua información referidas al ambiente. Proponer espacios para el cuidado y preservación del ambiente. Insertar el área del ambiente como parte de la transversalidad del currículo escolar. Realizar campañas continuas para el cuidado del agua.
A los estudiantes, cuidar y preservar el ambiente, participar en campañas ambientalistas, construir jardines y viveros escolares, reutilizar productos de gran impacto en el ambiente para asignarle nueva función.

A los guardianes y grupos ecológicos, ser vigilantes del cuidado del medio ambiente, participar en eventos referidos a la preservación del ecosistema, ser divulgadores del mensaje sobre el cuidado del medio ambiente.

\section{Referencias}

Conferencia Intergubernamental de Educación Ambiental de Tbilisi. (1977). Recuperado de: http://eaxxi.blogspot.com/2012/10/tbilisi1997-declaracion-de- la.html. (12/12/2018).

Flores, C. (2015). Educación ambiental en representaciones de docentes de escuelas secundarias. CPU - e. Revista de Investigación Educativa 16Veracruz, México: Universidad Veracruzana, 3. Obtenido de Recuperado de http://www.redalyc.org/articulo.oa?id=2831 $28328003(12 / 12 / 2018)$.

González, E. (2012). Formación de la conciencia ecológica como estructura compleja para la transformación educativa. Táchira, Venezuela: Lito Forma.

López, I. (2011). Medio Ambiente y PRAE. Bogotá: Universidad Nacional de Colombia.

Martínez, R. (2010). La importancia de la educación ambiental ante la problemática actual. Revista Electrónica Educare, vol. XIV, núm. 1, enero-junio, pp. 97-111.

Universidad Nacional Heredia, Costa Rica. Recuperado de:

https://www.redalyc.org/pdf/1 941/1941144 19010.pdf (12/12/2018).

Ministerio de Educación Nacional (2017). 
Proyecto ambiental educar para el desarrollo sostenible. PRAE Recuperado de: http://www.mineducacion.gov.c o/1621/arti cle-90893.html. (12/12/2018).

Ministerio de Educación Nacional y de Ambiente (2014.). Educar para el desarrollo sostenible. Recuperado de:

www.mineducacion.gov.co/162 1/article-9089 3.htm $(12 / 12 / 2018)$.

Navarro, L. (2007). Problemática de la educación ambiental en las instituciones educativas. México: Ibrahim.

Plaza, C. (2017). La importancia de la educación ambiental. Cuenca: Editorial EcoExperience.

Rivas, J. (2006). Proyecto educativo integrar comunitario para la dotación de áreas verdes a la Escuela Básica Nacional 12 de marzo. Tesis de grado. Universidad Rafael Urdaneta, Maracaibo, Venezuela.

Recuperado en:

https://www.redalyc.org/pdf/658/65828406 006.pdf (12/12/2018).

Sarango, J. Sánchez, S. y Landivar, J. (2016). Educación ambiental. ¿Por qué la Historia? Revista Universidad y Sociedad [seriada en línea], 8 (3). pp. 184 -187. Recuperado de: http://scielo.sld.cu/pdf/rus/v8 n3/rus25316. pdf $(12 / 12 / 2018)$.

UNESCO - PNUMA. (1990). Programa Internacional de Educación Ambiental. París: La Organización. Recuperado en: https://unesdoc.unesco.org/ark:/48223/pf00 00038550_spa (12/12/2018). 